Revue québécoise de psychologie

Revue ${ }^{\text {québécol̀se }}$

de psychologie

\title{
PORTRAIT PSYCHOLOGIQUE ET SOCIAL DE MÈRES INDIENNES \\ RÉFUGIÉES : LES ENJEUX LIÉS À L'ATTACHEMENT DE \\ L'ENFANT
}

\section{SOCIAL AND PSYCHOLOGICAL PROFILE OF INDIAN REFUGEE MOTHERS: CHALLENGES RELATED TO CHILD ATTACHMENT}

\section{Vanessa Lecompte, Zoé Richard-Fortier et Cécile Rousseau}

Volume 38, numéro 3, 2017

L’ACCUEIL DES RÉFUGIÉS : COMPLEXITÉ, RÉSILIENCE ET

CRÉATIVITÉ

REFUGEE HOME: COMPLEXITY, RESILIENCE AND CREATIVITY

URI : https://id.erudit.org/iderudit/1041838ar

DOI : https://doi.org/10.7202/1041838ar

Aller au sommaire du numéro

Éditeur(s)

Revue québécoise de psychologie

ISSN

2560-6530 (numérique)

Découvrir la revue

Citer cet article

Lecompte, V., Richard-Fortier, Z. \& Rousseau, C. (2017). PORTRAIT

PSYCHOLOGIQUE ET SOCIAL DE MÈRES INDIENNES RÉFUGIÉES : LES ENJEUX

LIÉS À L'ATTACHEMENT DE L'ENFANT. Revue québécoise de psychologie, 38(3),

61-78. https://doi.org/10.7202/1041838ar
Résumé de l'article

L'objectif de cet article utilisant une lunette qualitative à partir de mesures quantitatives est de dresser le portrait de quatre mères Indiennes réfugiées vivant un stress migratoire élevé et de présenter les conséquences liées à l'attachement de l'enfant. Les résultats indiquent des niveaux cliniques d'anxiété et de dépression ainsi qu'un soutien social variable selon la source de soutien. Chez l'enfant, la sécurité d'attachement est faible, il y a absence de comportements évitants et la prévalence de comportements ambivalents et désorganisés est élevée. Les résultats suggèrent que des approches qui misent sur un soutien de la dyade mère-enfant pourraient être appropriées pour ces femmes. 


\title{
PORTRAIT PSYCHOLOGIQUE ET SOCIAL DE MĖRES INDIENNES RÉFUGIÉES : LES ENJEUX LIÉS À L'ATTACHEMENT DE L'ENFANT
}

SOCIAL AND PSYCHOLOGICAL PROFILE OF INDIAN REFUGEE MOTHERS: CHALLENGES RELATED TO CHILD ATTACHMENT

\author{
Vanessa Lecompte ${ }^{1} \quad$ Zoé Richard-Fortier \\ Université McGill CIUSSS Centre-Sud-de-l'Île-de-Montréal \\ Cécile Rousseau \\ Université McGill
}

Les immigrants et réfugiés d'Asie du Sud (Bangladesh, Inde, Pakistan et Sri Lanka) représentent le plus grand groupe de migrants au Canada (Statistique Canada, 2011). Les études ont démontré que les immigrants de $1^{\text {re }}$ génération provenant d'Asie du Sud, particulièrement les femmes, sont à risque de stress migratoire et de problèmes de santé mentale (Ahmad et al., 2005). Selon la littérature, ce groupe spécifique semble à risque de développer un stress migratoire dû, entre autres, à la grande distance socioculturelle entre le pays d'accueil et le pays d'origine, aux nouvelles normes sociales et culturelles, à la barrière importante de la langue ainsi qu'aux déceptions possibles quant aux attentes liées à leur nouvelle vie après avoir quitté leur pays d'origine (Ahmad et al., 2005; Khan et Watson, 2005; Nilaweera, Doran et Fisher, 2014; Samuel, 2009). De plus, puisque la migration implique de laisser derrière des membres de la famille, des amis ou d'autres membres de la communauté, le réseau social se retrouve inévitablement réduit (Furnham et Shiekh, 1993). Dans une étude réalisée auprès de 447 réfugiés provenant de l'Asie, le stress postmigratoire, impliquant majoritairement le manque de soutien social, les pertes multiples (réseau social, statut économique) et l'adaptation à la nouvelle culture, prédisait davantage la santé mentale que le stress prémigratoire, caractérisé par les évènements traumatiques (Nicholson, 1997). En d'autres mots, le stress postmigratoire était le prédicteur le plus important de la santé mentale (anxiété, dépression, stress posttraumatique) après la migration, et ce, au-delà des évènements traumatiques vécus avant la migration, même si ceux-ci prédisaient également l'adaptation de l'individu. Pour plusieurs personnes réfugiées, ce stress lié à la migration peut être transitoire, mais peut aussi perdurer plusieurs années (Ellis, Murray et Barrett, 2014). Malgré le stress important que ces individus peuvent vivre, il est essentiel de noter que les personnes immigrantes et réfugiées ont moins tendance à chercher de l'aide ou à être référées aux services de santé mentale, même lorsque celles-ci présentent un niveau de détresse considérable (Guzder, Santhanam-Martin et

1. Adresse de correspondance : Département de Psychiatrie, Université McGill, 7085, rue Hutchison, Montréal (QC), H3N 1Y9. Téléphone : 514-273-3800, poste 6589. Courriel : vanessa.lecompte@mail.mcgill.ca 
Rousseau, 2014). Pour plusieurs réfugiés, la stigmatisation associée aux problèmes de santé mentale peut constituer une barrière pour chercher de l'aide (Osterman et De Jong, 2007). L'accès aux services psychosociaux constitue donc un enjeu pour cette population.

\section{Conséquences liées à la migration}

Une étude récente a démontré que les personnes réfugiées présentent généralement davantage de problèmes de santé mentale, tels que la dépression majeure, l'anxiété généralisée et le trouble de stress posttraumatique, que les personnes immigrantes non réfugiées (Fazel, Wheeler et Danesh, 2005; Murray, Davidson et Schweitzer, 2010). En plus des stresseurs liés à la migration, tels que l'exposition potentielle à la violence ou aux conditions de vie difficiles, les stresseurs postmigratoires sont considérables et peuvent engendrer des symptômes d'anxiété et de dépression. Les obstacles auxquels les familles doivent souvent faire face, en plus du désillusionnement relativement à la nouvelle vie, ont des effets néfastes sur l'adaptation de l'individu. En effet, l'incertitude quant au statut de réfugié, la perte d'un statut social, l'adaptation à la nouvelle culture et la perte du réseau social sont préjudiciables pour le bien-être émotionnel de l'individu et peuvent ainsi mener à des problèmes d'adaptation (Ahmad et al., 2005; Kirmayer et al., 2011). Évidemment, ce ne sont pas tous les réfugiés qui manifestent des symptômes importants d'anxiété ou de dépression. La vulnérabilité personnelle de l'individu ainsi que l'expérience pré et postmigratoire contribuent à son apparition (Bhugra et Ayonrinde, 2004). La perception de la société d'accueil face au groupe en question a également son rôle à jouer dans l'adaptation de l'individu. Dans le cas des migrants provenant d'Asie du Sud, l'attitude parfois contradictoire ou discriminante du pays d'accueil peut miner l'intégration de l'individu dans sa nouvelle société et favoriser la marginalisation (Abouguendia et Noels, 2001).

\section{Soutien social}

Le bien-être émotionnel de l'individu réfugié dépend non seulement de son expérience postmigratoire, mais également du cadre social dans lequel il évolue. En effet, certains facteurs psychosociaux, tel le soutien social, ont une influence sur la santé mentale soit en augmentant ou en diminuant la vulnérabilité de l'individu aux nombreux stresseurs environnementaux (Schweitzer, Melville, Steel et Lacherez, 2006). Considérant que les interactions sociales et l'identification au système culturel façonnent l'identité de l'individu, la perte de la famille élargie et du réseau social à la suite de la migration contribue à accentuer les symptômes d'anxiété et de dépression (Vahabi et Damba, 2015). Dans l'optique où les personnes réfugiées vivent très souvent des perturbations associées aux séparations avec l'unité familiale et au système culturel 
(Miller et al., 2002), ceci est particulièrement vrai dans le contexte des migrants d'Asie du Sud, où les différences entre la culture d'origine et la culture du pays d'accueil sont considérables. Conséquemment, promouvoir le soutien social en favorisant les rencontres intercommunautaires impliquant des membres de la communauté d'origine et des membres de la communauté d'accueil est d'une importance considérable afin de réduire le sentiment d'aliénation et d'encourager un sentiment d'appartenance qui contribue à la santé mentale de l'individu (Strang et Ager, 2010).

\section{Attachement de l'enfant}

Une conséquence importante du stress migratoire de la mère survient au niveau de la dyade mère-enfant, plus précisément sur le plan de la relation d'attachement. L'attachement se définit comme un lien affectif durable qu'un enfant forme avec un donneur de soins (Ainsworth, 1989). Lorsque le parent est sensible et réceptif aux besoins de l'enfant, celui-ci agit comme base de sécurité, ce qui permet à l'enfant d'explorer son environnement, tout en sachant que le parent est disponible en cas de besoin. L'enfant développe alors un attachement sécurisant, qui est connu comme étant le patron d'attachement le plus optimal pour le développement de l'enfant. L'enfant est confortable en présence du parent, les affects sont partagés et l'expression émotionnelle est ouverte. Cependant, lorsque le parent est peu sensible aux besoins de l'enfant, est inconsistant, rejetant ou même épeurant, l'enfant développe alors un attachement insécurisant, caractérisé soit par l'évitement, l'ambivalence ou la désorganisation. La qualité de la relation d'attachement est étroitement liée à la sensibilité du parent aux besoins de l'enfant (Moss et al., 2011b). Plus précisément, les enfants ayant un attachement évitant ont tendance à démontrer une neutralité affective ainsi qu'un évitement physique avec le parent. Les conversations sont peu fréquentes et axées sur le jeu ou les tâches plutôt que sur la relation. Les conversations ayant un contenu émotionnel ou personnel sont peu fréquentes. Les enfants ayant un attachement ambivalent démontrent généralement une expression émotionnelle exagérée, une dépendance et des comportements immatures ou résistants avec le parent. II n'est pas rare que l'enfant crie après le parent, entre en conflit avec celui-ci ou fasse des crises en présence du parent. L'enfant ayant un attachement ambivalent peut aussi paraitre fragile et présenter des comportements immatures pour son âge, tels que parler avec une petite voix ou mettre les mains dans la bouche. Quant à eux, les enfants ayant un attachement désorganisé présentent des comportements contradictoires ou incohérents en présence du parent, tels que des comportements successifs ou simultanés d'approche et d'évitement (p. ex., approcher le parent pour du réconfort et se retourner rapidement à l'approche de celui-ci) ou présentent de la confusion ou de l'appréhension à l'égard du parent ( $p$. ex., mettre la main au visage à 
l'approche du parent, expressions faciales de peur). Ces enfants ne peuvent utiliser la figure d'attachement comme source de protection et bien souvent la figure d'attachement est aussi source de peur. Ces observations sont faites lors de la Situation Étrange, mais sont également observables en milieu naturel, c'est-à-dire en dehors de la procédure de laboratoire (Moss et Lecompte, 2016). À l'âge préscolaire (à partir de 36 mois), environ $2 / 3$ des enfants qui présentaient des comportements désorganisés à la petite enfance présentent désormais des comportements contrôlants envers le parent, que ce soit de nature punitive ou attentionnée (Lyons-Ruth, Easterbrooks et Cibelli, 1997; Moss, Cyr et Dubois-Comtois, 2004). Ceci confirme l'hypothèse avancée par Aber et Allen (1987) voulant que les enfants étant dans une relation d'attachement désorganisée doivent porter une attention excessive à surveiller les comportements du parent au détriment des apprentissages et de l'exploration. La stratégie de contrôle punitif se manifeste par des comportements hostiles et directifs envers le parent et peut aussi se manifester par des menaces verbales ou des atteintes physiques. La stratégie de contrôle attentionné implique que l'enfant se soucie exagérément du parent en l'orientant, le guidant ou en l'encourageant pour le maintenir dans un état émotionnel stable (Moss, Bureau, St-Laurent et Tarabulsy, 2011a). II est connu qu'un attachement insécurisant, et plus particulièrement la désorganisation, place l'enfant sur une trajectoire développementale à risque (Lecompte et Moss, 2014; Moss, Rousseau, Parent, St-Laurent et Saintonge, 1998).

Le cadre théorique de l'attachement devient d'autant plus important avec les populations immigrantes ou réfugiées qui font souvent l'expérience de pertes multiples et de stress. L'état d'esprit du parent lié à l'attachement, par l'entremise par exemple de la dissociation, de l'impuissance, etc., relativement à ses expériences présentes et passées, teintera inévitablement les interactions avec l'enfant (Solomon et George, 2011). L'état d'esprit non résolu de la mère lié à l'attachement est un facteur critique qui influence la façon dont les expériences passées de pertes et d'abus affecteront négativement le rôle de parent (Jacobvitz, Leon et Hazen, 2006). Lorsque le parent a vécu des évènements traumatiques au cours de sa propre enfance ou même à l'âge adulte, l'état d'esprit découlant du trauma non résolu interfère directement avec les comportements parentaux (Hesse et Main, 2000). L'attachement de l'enfant est associé à la santé mentale du parent (anxiété, dépression) dans différentes cultures, et ce, peu importe le statut d'immigration (Emmen, Malda, Mesman, Ekmekci et van IJzendoorn, 2012; Grossmann, Grossmann et Keppler, 2005). Dans un contexte de migration, il est évident que les caractéristiques spécifiques à la culture d'origine vont aussi influencer la nature des interactions avec l'enfant (Keller, 2016). Cet aspect est non négligeable dans la transposition du modèle auprès de 
différentes cultures. Toutefois, il n'en demeure pas moins que lorsqu'il est question d'attachement, ce sont les comportements de l'enfant qui sont considérés et, l'enfant étant né ici, n'a pas connu autre chose que la relation dyadique avec le parent. Dans ce sens, la culture et les expériences présentes et passées du parent vont certainement expliquer les comportements de celui-ci envers l'enfant, mais l'enfant, lui, doit composer avec les comportements du parent tels qu'ils sont. L'enfant doit pouvoir se fier sur son parent pour obtenir du réconfort en cas de détresse et n'a pas nécessairement accès à la famille élargie comme cela aurait probablement été le cas dans le pays d'origine.

\section{Objectifs et hypothèses}

L'objectif principal de cette étude est de dresser un portrait de quatre mères Indiennes réfugiées vivant dans le quartier de Parc-Extension, à Montréal. Les objectifs spécifiques sont d'examiner, de manière qualitative descriptive, mais faisant usage de mesures quantitatives, les niveaux d'anxiété et de dépression, le soutien social perçu ainsi que l'attachement de l'enfant. II est attendu que ces mères et leur enfant présenteront plusieurs facteurs de vulnérabilité, c'est-à-dire des symptômes importants d'anxiété et de dépression, un soutien social faible et une faible sécurité d'attachement chez l'enfant. La santé mentale et le soutien social ont rarement été examinés relativement à l'attachement chez des familles réfugiées au Québec.

\section{MÉTHODOLOGIE}

\section{Participants}

Les participantes présentées dans cette étude sont quatre mères originaires de l'Inde et leur enfant. Deux des mères étaient enceintes au moment de leur arrivée au Canada et les deux autres ont eu leur enfant moins d'un an après leur arrivée au pays. Les mères ont toutes le statut de réfugiées et ont un revenu familial annuel sous le seuil de pauvreté (Gouvernement du Québec, 2014). Les caractéristiques sociodémographiques de chacune des mères sont présentées dans le Tableau 1.

\section{Procédure}

Les quatre participantes décrites dans cette étude font partie d'un projet de recherche plus large $(\mathrm{N}=40)$ toujours en cours visant à examiner les facteurs de risques qui influencent l'attachement de l'enfant dans une population vivant un stress migratoire élevé. Ces quatre mères ont été sélectionnées puisqu'elles ont toutes le statut de réfugiées, les autres étant 
Mères réfugiées et attachement de l'enfant

Tableau 1

Portrait sociodémographique des mères à l'étude

\begin{tabular}{|c|c|c|c|c|}
\hline Caractéristiques & Mère 1 & Mère 2 & Mère 3 & Mère 4 \\
\hline Âge de la mère & 30 à 39 ans & 30 à 39 ans & 20 à 29 ans & 20 à 29 ans \\
\hline Âge de l'enfant & 20 mois & 36 mois & 24 mois & 36 mois \\
\hline Genre de l'enfant & Fille & Garçon & Fille & Garçon \\
\hline Enfant né au Canada & Oui & Oui & Oui & Oui \\
\hline $\begin{array}{l}\text { Enfant fréquente la } \\
\text { garderie }\end{array}$ & Non & Oui & Oui & Non \\
\hline Pays d'origine de la mère & Inde & Inde & Inde & Inde \\
\hline $\begin{array}{l}\text { Nombre d'années au } \\
\text { Canada }\end{array}$ & 2 ans & 3 ans & 2 ans & 4 ans \\
\hline Revenu familial annuel & $-20000 \$$ & $-20000 \$$ & $\begin{array}{l}20,000 \$- \\
25,000 \$\end{array}$ & $-20000 \$$ \\
\hline $\begin{array}{l}\text { Niveau d'éducation } \\
\text { complété }\end{array}$ & Secondaire & Primaire & Universitaire & Universitaire \\
\hline Langue & Hindi & Punjabi & Punjabi & Punjabi \\
\hline Religion & Christianisme & Sikhisme & Hindouisme & Sikhisme \\
\hline Statut conjugal & Mariée & Mariée & Mariée & Mariée \\
\hline Emploi & Non & Non & Non & Non \\
\hline Autres enfants & Oui & Non & Non & Enceinte \\
\hline
\end{tabular}

citoyennes canadiennes ou résidentes permanentes. Le projet a initialement été présenté aux familles par une professionnelle de la santé travaillant au CLSC de Parc-Extension, à Montréal. Lorsque les familles ont accepté d'être contactées par l'équipe de recherche, un membre de l'équipe a communiqué avec celles-ci afin de leur expliquer la nature de leur implication et les objectifs généraux de l'étude. Les mères et leur enfant ont été rencontrés une seule fois lors d'une rencontre d'environ $1 \mathrm{~h} 30$ au domicile. Au moment de la rencontre, le consentement écrit a été obtenu pour chacune des mères. Les questionnaires ont été répondus en anglais avec ou sans la présence d'une interprète, tout dépendant du niveau de maitrise de l'anglais. Deux des quatre mères présentées dans cette étude ont bénéficié de la présence d'une interprète au moment de la rencontre.

\section{Instruments de mesure}

Le Questionnaire sociodémographique comprend des questions sur les aspects sociodémographiques de la mère et de sa famille (âge, genre, pays d'origine, langue, religion, revenu, éducation, statut conjugal, etc.). 
Le Hopkins Symptoms Check List (HSCL-25;Derogatis, Lipman, Rickels, Uhlenhuth et Covi, 1974) est un questionnaire complété par la mère qui comprend 25 questions. Cet instrument mesure la présence et le degré des symptômes d'anxiété et de dépression récents. Le participant répond aux énoncés en fonction d'une échelle Likert de 4 points allant de 1 (pas du tout) à 4 (énormément). Des exemples d'items sont «Vous sentir sans espoir face à l'avenir » et "Nervosité ou impression de tremblements intérieurs ». Ce questionnaire génère un score continu d'anxiété, de dépression, un score total ainsi que des scores cliniques (seuil clinique établi à 1,75 dans la littérature). Les échelles continues d'anxiété (alpha $=0,79$ ) et de dépression (alpha $=0,88$ ) ainsi que les scores cliniques sont utilisés dans ce projet. Ce questionnaire a été largement utilisé avec des populations d'Asie du Sud (p. ex., Sulaiman et al., 2014).

Le Multidimensional Scale of Perceived Social Support (MPSS; Zimet, Dahlem, Zimet et Farley, 1988) est un questionnaire comprenant 12 items qui vise à examiner la perception du soutien social provenant de différences sources, soit d'une personne significative, de la famille et des amis. Les choix de réponses vont de 1 (très fortement en désaccord) à 7 (très fortement en accord). Les réponses aux items génèrent trois échelles (personne significative, famille, amis) ainsi qu'un score total de soutien social. Une moyenne élevée sur les différentes échelles indique un niveau élevé de soutien social perçu. Des exemples d'items sont « Je reçois de ma famille toute l'aide émotionnelle et le soutien dont j'ai besoin » et " Je peux parler de mes problèmes avec mes amis ». Pour ce projet, les trois échelles, personnes significatives (alpha $=0,70)$, famille (alpha $=0,80$ ) et amis (alpha $=0,90)$, sont utilisées. Cet instrument présente de bonnes propriétés psychométriques transculturelles (Aroian, Templin et Ramaswamy, 2010).

Les comportements d'attachement de l'enfant ont été codifiés à partir d'une séquence de jeux libres filmée de 15 minutes. Aucune instruction spécifique n'est donnée à la mère, autre que de rester dans la pièce avec l'enfant et faire comme ils feraient normalement. Le chercheur attendait à l'extérieur de la pièce. La relation d'attachement se construit par les interactions avec la figure de soins dans un contexte naturel. Dans ce sens, l'observation de l'attachement dans un contexte plus écologique, c'est-à-dire sans le stress induit par la Situation Étrangère, a initialement été proposée par Ainsworth, Bell et Stayton (1971) puis reprise par Pederson et Moran $(1995,1996)$. Compte tenu de la vulnérabilité de la population étudiée, la codification de l'attachement en dehors de la Situation Étrangère a été privilégiée. La séquence filmée a été codifiée à l'aide du système Preschool and Early School-Age Attachment Rating Scales (PARS; Moss, Lecompte et Bureau, 2015). Ces échelles ont été 
développées à partir des systèmes largement utilisés de Cassidy et Marvin (1992) et Main et Cassidy (1988) pour codifier l'attachement de l'enfant entre 2 et 7 ans. L'utilisation de scores continus comme complément aux classifications permet davantage de puissance statistique avec de petits échantillons et génère un portrait plus représentatif de l'enfant en prenant en considération des indicateurs sur plusieurs dimensions plutôt qu'en " forçant » parfois une classification. L'utilisation des échelles nécessite, de la part du codeur, d'observer de quelle façon les stratégies d'attachement se manifestent par l'entremise de différentes modalités (p. ex., regards, affects, proximité) ainsi que d'observer comment ces modalités fonctionnent ensemble. L'intensité et la fréquence/durée des comportements sont considérées au moment d'assigner un score sur les différentes échelles. Les scores pour chacune des échelles vont de 1 (comportement absent) à 9 (très prévalent) avec un score de 5 représentant le seuil pour établir une classification (Moss et al., 2015). Des scores sont générés pour les 6 échelles suivantes: sécurité, évitement, ambivalence, désorganisation, contrôle punitif, contrôle attentionné. Les bandes ont été codifiées par un codeur expérimenté et l'accord interjuge a été établi sur un échantillon distinct. Puisque les mères parlaient leur langue maternelle avec l'enfant durant la séquence filmée, les bandes ont été traduites en anglais par une interprète. Ces échelles d'attachement ont été utilisées avec des populations immigrantes et non immigrantes (p. ex., Huber, McMahon et Sweller, 2015).

\section{RÉSULTATS}

\section{Prévalence de l'anxiété et de la dépression}

L'examen des scores révèle des niveaux élevés d'anxiété et de dépression, et ce, pour les quatre mères réfugiées de l'échantillon. Les moyennes (anxiété $=2,33$, dépression $=2,23$ ) sont situées au-dessus du seuil clinique (établi à 1.75). Plus précisément, une mère présente un score clinique d'anxiété uniquement et les trois autres mères présentent des scores cliniques d'anxiété et de dépression. Ces résultats sont corroborés par différentes manifestations lors des entretiens, tels que des pleurs fréquents, un sentiment d'être sans valeur mentionné à plusieurs reprises, des difficultés de concentration et, dans un des cas, des idées suicidaires. La Figure 1 présente les moyennes des scores d'anxiété et de dépression pour chacune des mères.

\section{Distribution des scores de soutien social}

Concernant la perception du soutien social, les moyennes des scores diffèrent selon la source du soutien. Bien qu'il existe des différences individuelles dans le soutien social perçu chez ces femmes, certaines 


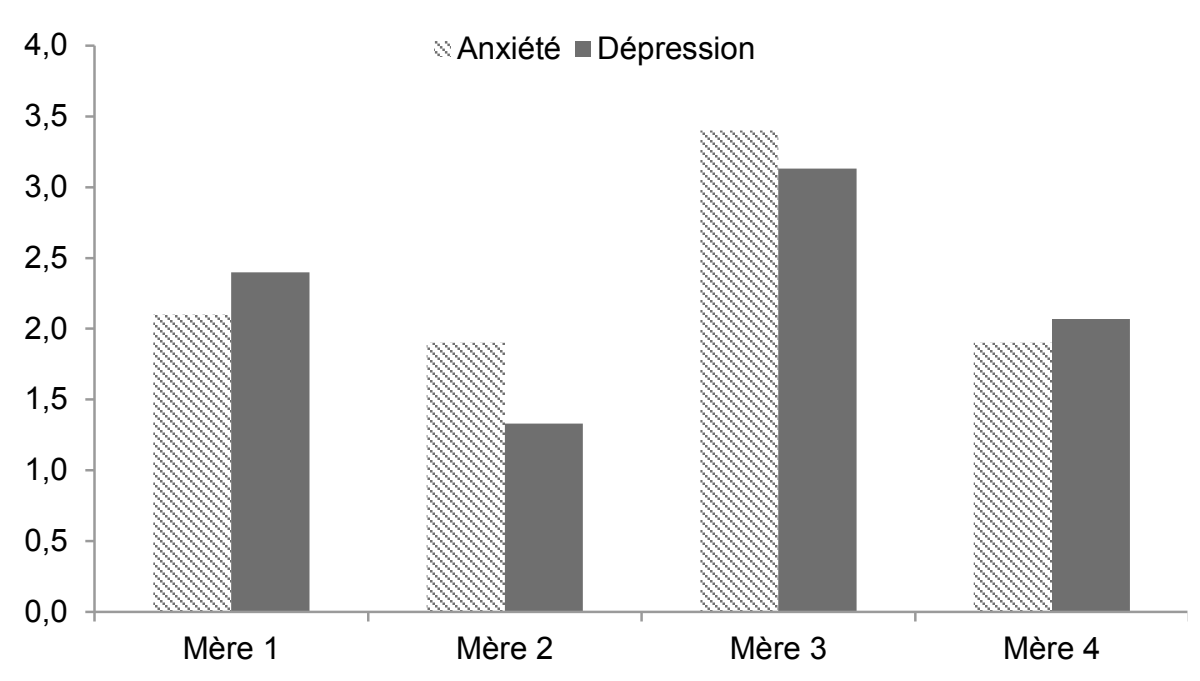

Figure 1. Moyennes des scores d'anxiété et de dépression. Seuil clinique fixé à 1,75 .

tendances semblent toutefois émerger. Pour la majorité d'entre elles, la source de soutien la plus importante est celle provenant d'une personne significative, identifiée dans tous les cas comme étant le mari. Le soutien social provenant de la famille est variable. Les familles habitent en Inde et les seuls contacts sont par téléphone. Les mères affirmaient toutes qu'elles évitaient de parler de leurs problèmes ou inquiétudes à leur famille en Inde, par peur de les inquiéter avec la précarité de leur situation. Les quatre mères affirmaient toutefois que le seul fait de parler à leur mère ou leur sœur par téléphone les faisait sentir moins seules. Finalement, le soutien social provenant des amis est plutôt faible. Ceci est corroboré par ce qui était évoqué durant l'entretien, c'est-à-dire que les mères affirmaient n'avoir aucun ami ici au Canada et ne parlent qu'à très peu de gens à l'extérieur de la maison. Les mères sont très soucieuses des commérages qui pourraient avoir lieu entre les membres de leur communauté, donc évitent d'entrer en relation avec qui que ce soit. Dans tous les discours, le sentiment d'isolement et de solitude était très présent et évoqué explicitement par chacune d'entre elles. La Figure 2 illustre la distribution des moyennes des scores de soutien social en fonction de la source de soutien pour chacune des mères.

\section{Distribution des scores d'attachement}

Une échelle de 1 à 9 est utilisée, puisque cet intervalle de scores fournit une large étendue d'intensité et de fréquence des comportements allant d'absents (1) à très prévalents (9) pour chacune des stratégies 


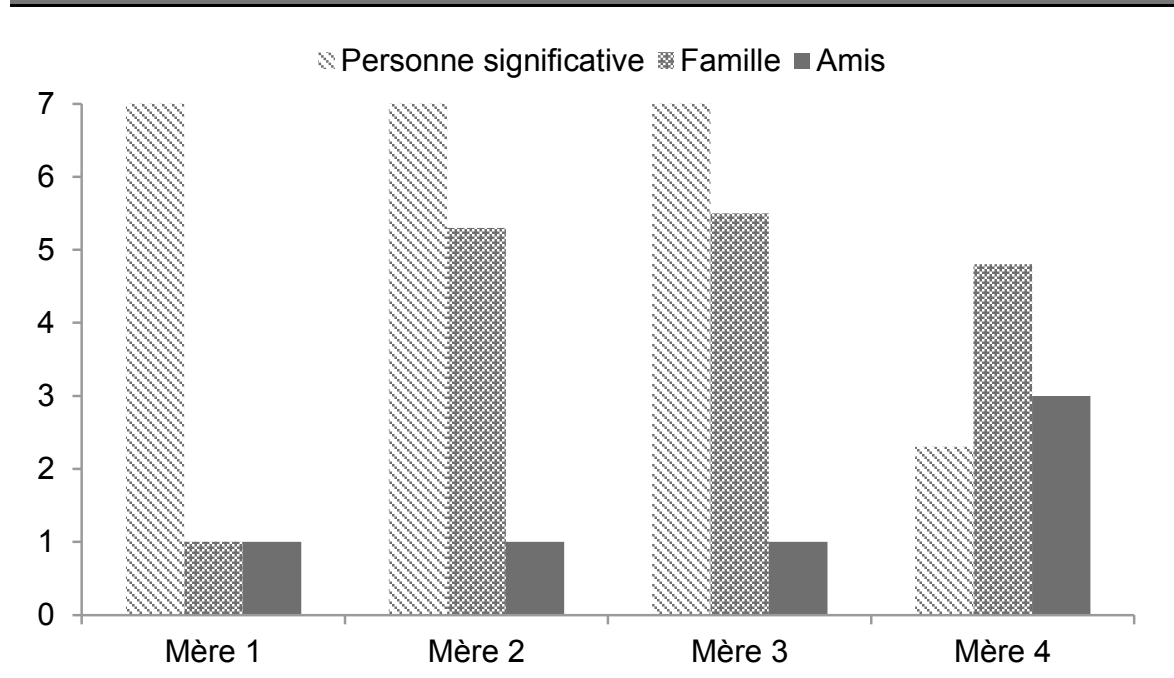

Figure 2. Moyenne des scores de soutien social perçu selon la source de soutien.

(p. ex., sécurité, évitement, etc.) avec un score de 5 représentant le seuil pour établir une classification (Moss et al., 2015). À la suite de l'examen de la distribution des scores d'attachement, il est possible d'établir certaines tendances présentes chez l'ensemble des enfants. En premier lieu, les scores sur l'échelle de sécurité indiquent un niveau de sécurité d'attachement très faible, et ce, pour tous les enfants (scores allant de 3 à 4.5), ce qui met en lumière les difficultés sur le plan de la sensibilité maternelle, étroitement liée à la sécurité d'attachement. Pour ce qui est des comportements d'évitement, aucun enfant ne présente de tels comportements, les scores étant tous de 1. Concernant l'échelle d'ambivalence, tous les enfants présentent des comportements d'ambivalence envers le parent, et ce, de façon assez importante (scores allant de 3.5 à 6). Finalement, concernant la désorganisation, 3 des 4 enfants présentent des comportements désorganisés, que ce soit au niveau de la désorganisation comportementale, du contrôle punitif ou du contrôle attentionné (scores allant de 2 à 7 ). En somme, cet échantillon d'enfants de mères réfugiées représente une population à haut risque au niveau de l'attachement, avec un faible niveau de sécurité et la présence notable de comportements ambivalents et désorganisés qui posent un risque pour le développement de l'enfant.

\section{DISCUSSION}

L'objectif de cet article est de faire part d'une réalité qui mérite une attention clinique particulière, à partir du portrait de quatre dyades mèreenfant. Cette étude est la première à dresser un portrait psychologique et 
social de mères réfugiées Indiennes et à mettre en lumière les comportements d'attachement de l'enfant chez ces familles vivant un stress migratoire élevé. Malgré le contexte social actuel, ces mères et leurs enfants restent bien souvent dans l'ombre, et leur histoire demeure méconnue. Pourtant, la situation de ces mères et leur enfant est loin d'être unique et représente la réalité de plusieurs familles réfugiées. L'adaptation de ces enfants est cruciale et, sans prévention, l'attachement insécurisant et les conséquences associées ( $p$. ex., problèmes intériorisés et extériorisés) peuvent possiblement devenir des problèmes de santé publique (Ayotte, Fournier et Riberdy, 2009). L'ampleur des difficultés observées est inquiétante et mérite une attention particulière.

Sommairement, les résultats indiquent, chez les mères, des niveaux d'anxiété et de dépression élevés. En décrivant leur situation, les mères abordaient systématiquement le désillusionnement quant à la migration ainsi que le peu d'espoir relativement au futur. Ceci teinte inévitablement les interactions avec l'enfant, la mère étant moins disponible psychologiquement pour répondre aux besoins de celui-ci. Sans exception, les mères ont toutes affirmé que sans leur enfant, leur vie serait sans importance et que l'enfant constitue leur seul espoir quant à l'avenir. Le bien-être de ces mères s'articule presque exclusivement autour de l'enfant, ce qui, sans le vouloir, met beaucoup de pression sur ce dernier. Dans ce sens, en plus d'agir sur le plan de la santé mentale du parent, une intervention ciblant la dyade spécifiquement est primordiale. Bien que la dimension du soutien social soit essentielle pour sortir ces mères de l'isolement, les patrons de communication et d'interactions dysfonctionnels doivent être adressés directement, en bas âge, afin de modifier la trajectoire développementale de l'enfant (Moss et al., 2011b). Dans le but de promouvoir la sécurité d'attachement, l'intervention à court terme visant à augmenter la prévisibilité, la cohérence et les pratiques "chaleureuses " du parent envers l'enfant s'avère des plus efficaces (BakermansKranenburg, van IJzendoorn et Juffer, 2003, 2005). Concrètement, compte tenu de la barrière de la langue et des différences culturelles, ces mères bénéficieraient non pas d'enseignements théoriques sur la sensibilité maternelle et sur les interactions parent-enfant, mais plutôt d'interventions privilégiant un intermédiaire visuel, tel que la rétroaction vidéo, afin de constater concrètement l'impact de certains comportements sur l'enfant. Par exemple, si la mère devient intrusive à un certain moment, la rétroaction vidéo permettrait de voir explicitement la réaction de l'enfant par rapport à de tels comportements. Ainsi, il est plus facile de miser sur les bons comportements qui engendrent des réactions positives chez l'enfant, qu'en adoptant des méthodes plus "académiques ». Cette méthode d'intervention dyadique a été utilisée avec différentes populations à risque, notamment avec des mères maltraitantes et leur enfant (Moss et 
al., 2014). Pour les raisons mentionnées plus haut, cette méthode d'intervention serait bien adaptée aux mères immigrantes.

Des études antérieures soulignent également l'importance de bâtir un réseau social avec les gens provenant de la société d'accueil, tout en gardant des contacts réguliers, lorsque possible, avec la famille et les amis laissés derrière dans le pays d'origine (Jasinskaja-Lahti, Liebkind, Jaakkola et Reuter, 2006). Bien que l'établissement d'un réseau de soutien social soit important pour l'adaptation de l'individu, ceci n'est pas toujours évident à réaliser (García, Ramírez et Jariego, 2002). En effet, les femmes décrites dans cet article ont un réseau social fragilisé. Elles identifient toutes leur mari comme source de support, mais ne peuvent généralement pas reposer sur un groupe d'amies ou sur leurs familles pour étayer leur souffrance liée aux multiples défis de leur parcours. En effet, les mères présentées dans cet article rapportent avoir quelques contacts avec la famille dans le pays d'origine, sans toutefois pouvoir aborder leurs difficultés émotionnelles et financières par crainte d'inquiéter la famille. Les mères rapportent également avoir très peu d'amis, voire aucun pour la majorité d'entre elles. Le soutien provient donc le plus souvent du mari. Ces résultats soulèvent l'importance de créer concrètement un espace de discussion informel pour ces mères d'origine sud-asiatique, où celles-ci peuvent se rencontrer, discuter, cuisiner et ainsi tisser des liens avec d'autres mères vivant une situation similaire. De tels espaces de rencontre existent à l'heure actuelle, mais l'enjeu demeure toujours la mobilisation des mères vers ces lieux de rencontre.

Chez les enfants, un niveau de sécurité d'attachement très faible est observé, avec des comportements dénotant de l'ambivalence ou de la désorganisation. II est intéressant de souligner qu'aucun enfant ne présente d'indice comportemental d'évitement, dénotant, au sein de l'échantillon, une tendance de recours à des stratégies caractérisées par l'amplification de l'importance accordée à la relation, au détriment de l'exploration. Un parallèle observable apparait ainsi se dresser entre la difficulté de ces enfants à découvrir leur monde et celle de leur mère, isolée socialement et éprouvée psychologiquement. Des comportements d'attachement désorganisés sont aussi observables chez l'enfant. Bien que l'état d'esprit de la mère lié à l'attachement n'ait pas été directement mesuré dans cette étude, il est tout de même possible d'observer que les enfants ayant des comportements désorganisés ont des mères anxieuses ou dépressives. L'insensibilité du parent, les comportements parentaux épeurants et atypiques sont associés à la désorganisation chez l'enfant (Madigan et al., 2006). Bien que cela n'ait pas été empiriquement mesuré dans ce projet, il est possible de croire que les niveaux élevés d'anxiété et de dépression affectent la disposition psychologique de la mère à l'égard des interactions avec l'enfant. La validation de cette hypothèse auprès de 
cette population serait d'intérêt lors de recherches futures. En somme, force est de constater que la santé mentale de la mère et l'attachement de l'enfant apparaissent interreliés dans un parcours de vie éprouvé par des pertes et des séparations migratoires.

L'ensemble de nos observations amène à présenter la dyade comme unité d'intervention à privilégier où l'accompagnement de l'enfant et de la mère sont intégrés afin de renforcer un potentiel relationnel au service du développement de l'enfant (Moss et al., 2011b). Dans le même sens, notons qu'aucune des mères de l'étude ne reçoit de services en réponse à leurs symptômes anxieux ou dépressifs ou mentionne vouloir en faire la demande au moment de la collecte de données. Les services en petite enfance semblent ainsi représenter une passerelle d'accès permettant de rejoindre ces mères afin de proposer une démarche sensible à leurs propres besoins psychosociaux, et ce, grâce au levier puissant du dévouement qu'ont ces mères envers leur enfant. Dès lors, leur santé mentale ainsi que la sensibilité maternelle liée à la sécurité d'attachement deviennent toutes deux des cibles d'interventions prioritaires, considérant la détresse psychologique de ces dernières, l'impact sur l'attachement de l'enfant et ses implications développementales. La qualité de la relation d'attachement est dépendante de la sensibilité du donneur de soins aux besoins de l'enfant (Ainsworth, Blehar, Waters et Wall, 1978; Moss et al., 2011a). Dans cette optique, la mère et la relation deviennent les principaux piliers pour répondre aux défis qui pourraient se présenter dans l'évolution de l'enfant, au-delà des services qui pourraient lui être offerts directement. L'état d'esprit autonome de la mère lié à l'attachement, c'est-à-dire lorsque les expériences traumatiques sont résolues, ainsi que la sensibilité de la mère sont liés à la sécurité d'attachement théoriquement et empiriquement (Atkinson et al., 2005). Soulignons que cette progression s'édifie d'abord sur la base de sécurité qui peut être offerte à la mère au moyen des interventions et des ressources communautaires accessibles. De plus, cet accompagnement prend une couleur particulière dans un contexte migratoire précaire. Les liens tissés au sein de la société d'accueil s'inscrivent dans un processus d'intégration qui soutient les capacités d'exploration de la mère et, par le fait même, celles de son enfant.

Les résultats doivent être interprétés à la lumière de certaines limites. Dans un premier temps, les mères décrites dans cet article sont des mères originaires de l'Inde et présentent des caractéristiques sociodémographiques similaires. Dans ce sens, la prudence est de mise dans la généralisation des conclusions de cet article à d'autres populations réfugiées. Également, la taille de l'échantillon limite la généralisation des résultats. Bien que cela ne faisait pas partie des objectifs de l'étude, de l'information sur l'histoire prémigratoire de la mère n'a pas systématiquement été recueillie et les participantes n'ont pas toujours 
divulgué de telles informations. Conséquemment, les expériences passées potentiellement traumatiques n'ont pas été abordées. Finalement, bien que les scores d'attachement soient un indicateur important de la sensibilité maternelle (p. ex., score élevé sur l'échelle de sécurité d'attachement correspond à un parent plutôt sensible), aucune mesure de sensibilité maternelle n'a été utilisée dans ce projet. Les interprétations touchant à la sensibilité maternelle sont donc faites à partir des scores sur les échelles d'attachement et non à partir d'une mesure explicite de sensibilité maternelle.

\section{Conclusion}

L'examen qualitatif des données quantitatives portant sur quatre mères Indiennes réfugiées a permis de mettre en lumière les difficultés importantes auxquelles certaines mères font face en situation postmigratoire. Les niveaux élevés d'anxiété et de dépression ainsi qu'un réseau social faible dans le pays d'accueil constituent une réalité somme toute inquiétante. Les répercussions négatives sur le plan de la relation parent-enfant sont bien présentes et méritent une attention particulière. La situation de ces mères est loin d'être unique, et correspond à la situation de plusieurs mères réfugiées vivant à Montréal, notamment. Les recherches futures devraient considérer répliquer le devis auprès de mères réfugiées d'autres origines, afin de déterminer si ce portrait correspond aussi à celui d'autres cultures. Le suivi longitudinal de ces mères serait également important pour examiner l'évolution des symptômes, et ce, avec et sans intervention. À la lumière des résultats obtenus, il convient de valoriser les initiatives ancrées dans les milieux de vie permettant des rencontres entre les mères dans un contexte sécurisant, en plus de renforcer les ponts entre les offres de services au niveau de la santé mentale adulte et de la petite enfance. L'accompagnement de la mère et de son enfant peuvent ainsi se rejoindre de façon cohérente afin de faire écho au besoin, d'autant plus prégnant dans un contexte migratoire précaire, d'être portée par une communauté qui reconnait et valorise leur potentiel relationnel fondamental. Ces mères présentent des forces qui méritent d'être soulignées et valorisées, pour les renforcer dans leur rôle maternel.

\section{RÉFÉRENCES}

Aber, J. L. et Allen, J. P. (1987). Effects of maltreatment on young children's socioemotional development: An attachment theory perspective. Developmental Psychology, 23(3), 406414.

Abouguendia, M. et Noels, K. A. (2001). General and acculturation-related daily hassles and psychological adjustment in first-and second-generation South Asian immigrants to Canada. International Journal of Psychology, 36(3), 163-173.

Ahmad, F., Shik, A., Vanza, R., Cheung, A. M., George, U. et Stewart, D. E. (2005). Voices of South Asian women: immigration and mental health. Women \& Health, 40(4), 113-130. 
Ainsworth, M. D. S. (1989). Attachments beyond infancy. American Psychologist, 44(4), 709716.

Ainsworth, M. D. S., Bell, S. M. et Stayton, D. J. (1971). Individual differences in strangesituation behaviour of one-year olds: The origins of human social relations. Londres, Royaume-Uni : Academic Press.

Ainsworth, M. D. S., Blehar, M. C., Waters, E. et Wall, S. N. (1978). Patterns of attachment : A psychological study of the strange situation. Hillsdale, NJ: Erlbaum.

Aroian, K., Templin, T. N. et Ramaswamy, V. (2010). Adaptation and Psychometric Evaluation of the Multidimensional Scale of Perceived Social Support for Arab Immigrant Women. Health Care for Women International, 31(2), 153-169.

Atkinson, L., Goldberg, S., Raval, V., Pederson, D., Benoit, D., Moran, G.,... Leung, E. (2005). On the relation between maternal state of mind and sensitivity in the prediction of infant attachment security. Developmental Psychology, 41(1), 42-53.

Ayotte, V., Fournier, M. et Riberdy, H. (2009). La détresse psychologique des enfants et des adolescents montréalais... l'expression de différentes réalités? Montréal, QC : Direction de santé publique, Agence de la santé et des services sociaux de Montréal.

Bakermans-Kranenburg, M. J., van IJzendoorn, M. H. et Juffer, F. (2003). Less is more: Metaanalyses of sensitivity and attachment interventions in early childhood. Psychological Bulletin, 129(2), 195-215.

Bakermans-Kranenburg, M. J., van IJzendoorn, M. H. et Juffer, F. (2005). Disorganized infant attachment and preventive interventions: A review and meta-analysis. Infant Mental Health Journal, 26(3), 191-216.

Bhugra, D. et Ayonrinde, O. (2004). Depression in migrants and ethnic minorities. Advances in Psychiatric Treatment, 10(1), 13-17.

Cassidy, J. et Marvin, R. S. (1992). Attachment organization in three- and four-year-olds: Procedures and coding manual. Document inédit. Charlottesville, VA: University of Virginia.

Derogatis, L. R., Lipman, R. S., Rickels, K., Uhlenhuth, E. H. et Covi, L. (1974). The Hopkins Symptom Checklist (HSCL): A self-report symptom inventory. .Systems Research and Behavioral Science, 19(1), 1-15.

Ellis, B. H., Murray, K. et Barrett, C. (2014). Understanding the mental health of refugees: trauma, stress, and the cultural context. Dans R. Parekh (dir.) The Massachusetts General Hospital Textbook on Diversity and Cultural Sensitivity in Mental Health (p. 165187). New York, NY : Humana Press.

Emmen, R. A. G., Malda, M., Mesman, J., Ekmekci, H. et van IJzendoorn, M. H. (2012). Sensitive parenting as a cross-cultural ideal : Sensitivity beliefs of Dutch, Moroccan, and Turkish mothers in the Netherlands. Attachment \& Human Development, 14(6), 601-619.

Fazel, M., Wheeler, J. et Danesh, J. (2005). Prevalence of serious mental disorder in 7000 refugees resettled in western countries: a systematic review. The Lancet, 365(9467), $1309-1314$.

Furnham, A. et Shiekh, S. (1993). Gender, generational and social support correlates of mental health in Asian immigrants. International Journal of Social Psychiatry, 39(1), 2233.

García, M. F. M., Ramírez, M. G. et Jariego, I. M. (2002). Social support and locus of control as predictors of psychological well-being in Moroccan and Peruvian immigrant women in Spain. International Journal of Intercultural Relations, 26(3), 287-310.

Gouvernement du Québec. (2014). Seuil de faible revenu (avant impôt) pour chaque année selon la taille du ménage. Repéré à http://cdn.carra.gouv.qc.ca/G\%C3\%A9n\%C3\%A9ral/Pages/IN99KXXX00A001.aspx.

Grossmann, K. E., Grossmann, K. et Keppler, A. (2005). Universal and culture-specific aspects of human behavior: The case of attachment. Hove, Royaume-Uni: Erlbaum.

Guzder, J., Santhanam-Martin, R. et Rousseau, C. (2014). Gender, power and ethnicity in cultural consultation: Encountering the other in mental health care. Dans L. J. Kirmayer, J. Guzder et C. Rousseau (dir.), Cultural consultation (p. 163-182). New York, NY: Springer. 
Hesse, E. et Main, M. (2000). Disorganized infant, child, and adult attachment: Collapse in behavioral and attentional strategies. Journal of the American Psychoanalytic Association, 48(4), 1097-1127.

Huber, A., McMahon, C. A. et Sweller, N. (2015). Efficacy of the 20-week circle of security intervention: changes in caregiver reflective functionning, representations, and child attachment in an Australian clinical sample. Infant Mental Health Journal, 36(6), 556574.

Jacobvitz, D., Leon, K. et Hazen, N. (2006). Does expectant mothers' unresolved trauma predict frightened/frightening maternal behavior? Risk and protective factors. Development and Psychopathology, 18(2), 363-379.

Jasinskaja-Lahti, I., Liebkind, K., Jaakkola, M. et Reuter, A. (2006). Perceived discrimination, social support networks, and psychological well-being among three immigrant groups. Journal of Cross-Cultural Psychology, 37(3), 293-311.

Keller, H. (2016). Attachment. A pancultural need but a cultural construct. Current Opinion in Psychology, 8, 59-63.

Khan, S. et Watson, J. C. (2005). The Canadian immigrantion experiences of Pakistani women: Dreams confront reality. Counselling Psychology Quarterly, 18(4), 307-317.

Kirmayer, L. J., Narasiah, L., Munoz, M., Rashid, M., Ryder, A. G., Guzder, J.,... Pottie, K. (2011). Common mental health problems in immigrants and refugees: general approach in primary care. Canadian Medical Association Journal, 183(12), E959-E967.

Lecompte, V. et Moss, E. (2014). Disorganized and controlling patterns of attachment, role reversal, and caregiving helplessness: Links to adolescents' externalizing problems. American Journal of Orthopsychiatry, 84(5), 581-589.

Lyons-Ruth, K., Easterbrooks, M. A. et Cibelli, C. D. (1997). Infant attachment strategies, infant mental lag, and maternal depressive symptoms: Predictors of internalizing and externalizing problems at age 7. Developmental Psychology, 33(4), 681-692.

Madigan, S., Bakermans-Kranenburg, M. J., van IJzendoorn, M. H., Moran, G., Pederson, D. R. et Benoit, D. (2006). Unresolved states of mind, anomalous parental behavior, and disorganized attachment: A review and meta-analysis of a transmission gap. Attachment \& Human Development, 8(2), 89-111.

Main, M. et Cassidy, J. (1988). Categories of response to reunion with the parent at age 6: Predictable from infant attachment classifications and stable over a 1-month period. Developmental Psychology, 24(3), 415-426.

Miller, K. E., Weine, S. M., Ramic, A., Brkic, N., Bjedic, Z. D., Smajkic, A.,... Worthington, G. (2002). The relative contribution of war experiences and exile-related stressors to levels of psychological distress among Bosnian refugees. Journal of Traumatic Stress, 15(5), 377-387.

Moss, E., Bureau, J.-F., St-Laurent, D. et Tarabulsy, G. M. (2011a). Understanding disorganized attachment at preschool and school age: Examining divergent pathways of disorganized and controlling children. Dans J. Solomon et C. George (dir.), Disorganized attachment and caregiving (p. 52-79). New York, NY: Guilford Press.

Moss, E., Cyr, C. et Dubois-Comtois, K. (2004). Attachment at early school age and developmental risk: examining family contexts and behavior problems of controllingcaregiving, controlling-punitive, and behaviorally disorganized children. Developmental Psychology, 40(4), 519-532.

Moss, E., Dubois-Comtois, K., Cyr, C., Tarabulsy, G., St-Laurent, D. et Bernier, A. (2011b). Efficacy of a home-visiting intervention aimed at improving maternal sensitivity, child attachment, and behavioral outcomes for maltreated children: A randomized control trial. Development and psychopathology, 23(1), 195-210.

Moss, E. et Lecompte, V. (2016). Attachment observation for preschool and school-age period. Document inédit.

Moss, E., Lecompte, V. et Bureau, J. (2015). Preschool and early school-age attachment rating scales (PARS). Document inédit.

Moss, E., Rousseau, D., Parent, S., St-Laurent, D. et Saintonge, J. (1998). Correlates of attachment at school age: Maternal reported stress, mother-child interaction, and behavior problems. Child Development, 69(5), 1390-1405. 
Moss, E., Tarabulsy, G. M., St-Georges, R., Dubois-Comtois, K., Cyr, C., Bernier, A.,... Lecompte, V. (2014). Video-feedback intervention with maltreating parents and their children: Program implementation and case study. Attachment \& Human Development, 16(4), 329-342.

Murray, K. E., Davidson, G. R. et Schweitzer, R. D. (2010). Review of refugee mental health interventions following resettlement: best practices and recommendations. American Journal of Orthopsychiatry, 80(4), 576-585.

Nicholson, B. L. (1997). The influence of pre-emigration and postemigration stressors on mental health: A study of Southeast Asian refugees. Social Work Research, 21(1), 1931.

Nilaweera, I., Doran, F. et Fisher, J. (2014). Prevalence, nature and determinants of postpartum mental health problems among women who have migrated from South Asian to high-income countries: A systematic review of the evidence. Journal of Affective Disorders, 166, 213-226.

Osterman, J. E. et de Jong, J. T. V. M. (2007). Cultural issues and trauma. Dans M. J. Friedman, T. M. Keane et P. A. Resick (dir.), Handbook of PTSD: Science and Practice (p. 425-446). New York, NY: Guilford Press.

Pederson, D. R. et Moran, G. (1995). A categorical description of infant-mother relationships in the home and its relation to Q-sort measures of infant-mother interaction. Monographs of the Society for Research in Child Development, 60(2-3), 111-132.

Pederson, D. R. et Moran, G. (1996). Expressions of the attachment relationship outside of the Strange Situation. Child Development, 67(3), 915-927.

Samuel, E. (2009). Acculturative Stress : South Asian immigrant women's experiences in Canada's Atlantic Provinces. Journal of Immigrant \& Refugee Studies, 7(1), 16-34.

Schweitzer, R., Melville, F., Steel, Z. et Lacherez, P. (2006). Trauma, post-migration living difficulties, and social support as predictors of psychological adjustment in resettled Sudanese refugees. Australian and New Zealand Journal of Psychiatry, 40(2), 179-188.

Solomon, J. et George, C. (2011). Disorganized attachment and caregiving. New York, NY : Guilford Press.

Statistique Canada (2011). Immigration et diversité ethnoculturelle au Canada. Enquête nationale auprès des ménages. Repéré à http://www12.statcan.gc.ca/nhs-enm/2011/assa/99-010-x/99-010-x2011001-fra.pdf.

Strang, A. et Ager, A. (2010). Refugee integration: Emerging trends and remaining agendas. Journal of Refugee Studies, 23(4), 589-607.

Sulaiman, A. H., Bautista, D., Liu, C.-Y., Udomratn, P., Bae, J. N., Fang, Y.,... et Rush, A. J. (2014). Differences in psychiatric symptoms among Asian patients with depression: A multi-country cross-sectional study. Psychiatry and Clinical Neurosciences, 68(4), 245254.

Vahabi, M. et Damba, C. (2015). A feasibility study of a culturally and gender-specific dance to promote physical activity for South Asian immigrant women in the greater Toronto area. Women's Health Issues, 25(1), 79-87.

Zimet, G. D., Dahlem, N. W., Zimet, S. G. et Farley, G. K. (1988). The Multidimensional Scale of Perceived Social Support. Journal of Personality Assessment, 52(1), 30-41.

\section{RÉSUMÉ}

L'objectif de cet article utilisant une lunette qualitative à partir de mesures quantitatives est de dresser le portrait de quatre mères Indiennes réfugiées vivant un stress migratoire élevé et de présenter les conséquences liées à l'attachement de l'enfant. Les résultats indiquent des niveaux cliniques d'anxiété et de dépression ainsi qu'un soutien social variable selon la source de soutien. Chez l'enfant, la sécurité d'attachement est faible, il y a absence de comportements évitants et la prévalence de comportements ambivalents et désorganisés est élevée. Les résultats suggèrent que des approches qui misent sur un soutien de la dyade mère-enfant pourraient être appropriées pour ces femmes. 
Mères réfugiées et attachement de l'enfant

\section{MOTS CLÉS}

anxiété, dépression, soutien social, attachement, réfugiés

\section{ABSTRACT}

The objective of this article that uses a qualitative approach from quantitative measures is to describe the profile of four Indian refugee mothers experiencing high migration stress, and to present the challenges related to child attachment. Results indicate clinical levels of anxiety and depression, as well as variable social support depending on the source of support. Child attachment security is low, avoidant attachment behaviors are absent, and the prevalence of ambivalent and disorganized attachment behaviors is high. Results suggest that approaches that focus on supporting mother-child dyads would be appropriate for these women.

\section{KEY WORDS}

anxiety, depression, social support, attachment, refugees 